\title{
Mechanochemical Dechlorination of PVC by Utilizing Eggshell Waste Acta Physica Polonica A 126, 884 (2014), ERRATUM
}

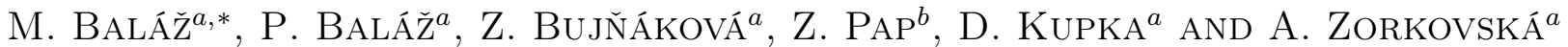 \\ ${ }^{a}$ Institute of Geotechnics, Slovak Academy of Sciences, Košice, Slovakia \\ ${ }^{b}$ Institute of Raw Material Preparation and Environmental Processing, University of Miskolc, Miskolc, Hungary \\ Within this work, the dechlorination of polyvinylchloride (PVC) chemical as a model by co-milling with \\ eggshell was performed in a planetary ball mill in order to show that mechanochemistry can be utilized for the \\ simultaneous treatment of two wastes, while the products of the treatment can be used in further applications. \\ The products of the reaction are water-soluble calcium chloride $\left(\mathrm{CaCl}_{2}\right)$, which can be used e.g. for de-icing of \\ roads in winter and organic residue on the basis of ethylene, which can be recovered as energy source. The highest \\ dechlorination yield (almost 97\%) was achieved under following milling conditions: molar ratio between Ca and \\ Cl: 2.34, ball-to-powder ratio: 65 , rotation speed of the planet carrier: $550 \mathrm{rpm}$, milling time: $4 \mathrm{~h}$ and material \\ of milling media: tungsten carbide. The optimum conditions were then applied for the removal of chlorine from \\ industrial waste - the abandoned PVC window parapet. In this case, 95\% dechlorination was evidenced.
}

original DOI: 10.12693/APhysPolA.126.884

actual DOI: 10.12693/APhysPolA.134.1247

PACS/topics: $99.10 . \mathrm{Cd}$

This article was originally published on October 2014 with an incorrect value of one parameter. Therefore second item in last line of the Table should read 5.06 instead of 2.35 and fifth paragraph on page 886, left column, should read:

From all the experiments, the experiment 8 was selected as optimum and therefore for the dechlorination of window parapet (exp. WP), the conditions used in this experiment were applied, with the exception of Ca:Cl molar ratio which was increased to 5.06. This is because the mass of powdered parapet containing much less chlorine was the same as the mass of pure PVC in experiment 8.

The authors apologize for this error.

*corresponding author; e-mail: balazm@saske.sk 